\title{
Instrument development and validation of the stroke pre-hospital delay behavior intention scale in a Chinese urban population
}

Qiuli Zhao ${ }^{1 *+}$, Li Yang ${ }^{1 \dagger}$, Qingqing Zuo ${ }^{1+}$, Xuemei Zhu' ${ }^{1}$ Xiao Zhang ${ }^{1}$, Yanni Wu' ${ }^{2}$ Liu Yang ${ }^{1}$, Wei Gao ${ }^{1}$ and Minghui Li ${ }^{1}$

\begin{abstract}
Background: Several stroke impairment scales are currently available for stroke patients but none of them provide information regarding the pre-stroke behavioral intentions of high-risk stroke patients and their relatives. This study's objective was to generate and validate a new written tool, the Stroke Pre-hospital Delay Behavior Intention (SPDBI) scale. It is suitable for use with high-risk stroke patients and their relatives to predict the likelihood of pre-hospital delay.

Methods: From a review of related studies, we formulated a prototype scale. We interviewed ten stroke patients in a semi-structured iterative process that included interviews with experts, high-risk patients, and their family members. Then, we pretested and filtered items. We next used a large sample size and factor analysis to determine the scale's structure. Finally, we checked the reliability and validity of the scale.
\end{abstract}

Results: We identified five sub-domains (stroke warning signs, non-treatment justification, symptom attributions, habitual response style, and emergency system use). The SPDBI demonstrated good internal consistency and test-retest reliability (Cronbach's a $=0.808$; Intraclass Correlation Coefficient $[I C C]=0.797$ ).

Conclusions: This SPDBI scale is a reliable, and valid measure of the likeliness of pre-hospital delay in high-risk stroke patients and their family members. It may provide scientific assessment for targeted health education intervention.

Keywords: Pre-hospital delay, Behavioral intention, High-risk stroke patients

\section{Background}

Stroke is the third leading cause of death in the United States, Canada, Europe, and Japan [1]. In China, cerebrovascular disease has become the leading cause of mortality and morbidity in both urban and suburban populations with 1,500,000-2,000,000 new strokes each year [1]. Many studies have demonstrated that thrombolytic therapy is only effective within the first 4.5 hours after the onset of an ischemic stroke [2,3]. Early intensive blood pressurelowering treatment is clinically feasible, well tolerated, and appears to reduce hematoma growth in acute intracerebral hemorrhage (ICH) [4]. People need to access emergency

\footnotetext{
* Correspondence: zhaoqiuli1957@163.com

${ }^{\dagger}$ Equal contributors

${ }^{1}$ School of Nursing, The 2nd Affiliated Hospital of Harbin Medical University, Harbin Medical University, 246 Xuefu Road, Harbin, HeiLongJiang Province

150086, China

Full list of author information is available at the end of the article
}

medical services quickly when early stroke symptoms occur. However, in most communities, only $1-7 \%$ of stroke victims arrive at the hospital in time for stroke revascularization therapies [1]. Moreover, in China, only $37 \%$ of patients were sent to the hospital within the effective therapeutic time window for strokes, and the rate of thrombolytic therapy for an ischemic stroke is estimated at only $1-3 \%$ [5]. In addition, more than $80 \%$ of Chinese stroke patients suffer their first stroke symptoms at home [5].

Pre-hospital delay time is the time from symptom onset to hospital arrival [6]. The delay occurs for numerous reasons; for instance, many patients and their relatives do not think their symptoms are serious, and a wait-and-see approach is adopted before seeking treatment. A serious stroke makes patients unable to ask for help themselves [7]. These pre-hospital delay behavioral intentions increase 
pre-hospital delay time and decrease the therapeutic efficacy of patient interventions.

The stroke impairment scales with warning signs were developed to quickly assess stroke patients by the public and/or paramedics, and include scales such as the Cincinnati Pre-Hospital Stroke Scale (CPSS), the Los Angeles Pre-hospital Stroke Screen (LAPSS), and the Face Arm Speech Test (FAST) [8-10]. These scales contain items about stroke symptoms, but simple recognition of specific stroke symptoms may not be associated with reduced prehospital delay $[11,12]$. Additionally, the Stroke Action Test (STAT) [13] evaluates the public's reaction to a stroke. The scores of the STAT predict the actions that the public would take if stroke symptoms occur, but cannot specifically ascertain the pre-hospital delay in behavioral intention.

Stroke pre-hospital delay time has increasingly become the focus of researchers' attention. Most studies have focused on determining time from patient stroke onset to hospital registration, and then judging whether there is a delay [14-17]. However, they have not addressed decreasing that delay. New tools capable of detecting the participant's behavioral intention if stroke symptoms occur and then assessing the possibility of a pre-hospital delay are needed.

Therefore, we developed a scale named the Stroke Pre-Hospital Delay Behavior Intention (SPDBI) scale, which includes not only stroke warning signs but also specific items on how the participants would think about or react to stroke signs in order to reduce stroke pre-hospital delays. It is intended to provide a scientific assessment tool for targeted health education intervention.

\section{Methods}

The study protocol was approved by the ethics committee of the 2nd Affiliated Hospital of Harbin Medical University. All of the experts and participants signed the informed consent form, were provided with a verbal explanation about the purpose of the study, and informed that participation was voluntary, confidential, and anonymous.

\section{Samples}

Using convenience sampling, we advertised for and telephoned participants who agreed to participate in this study from the 2nd Affiliated Hospital of Harbin Medical University and local community groups in Harbin City, China. The criteria for the participants were (1) high-risk stroke patients with a confirmed diagnosis of diabetes, coronary artery disease, hypertension, or hyperlipidemia; or (2) family members or those who might be responding to the stroke symptoms (e.g., partners, adult children, other family members, friends, or caregivers. Participants were volunteers 18 years of age or older. Participants were excluded if they had any of the following characteristics: (1) history of stroke, dementia, or severe psychiatric disorders; (2) altered consciousness; (3) deafness or blindness; or (4) were medical practitioners. Participants were in the hospital unit or invited to visit the community service center to complete the scale, which was accompanied by a short questionnaire about their personal details.

Initially, we included 30 experts for the preliminary scale. The expert criteria were medical doctors or nurses who had been engaged in related work for more than 10 years (from 6 neurology departments, 3 neurosurgery departments, and 1 emergency department). Then, 20 stroke high-risk patients and their family members who met the patient criteria were also invited from a geriatric department. A total of 312 eligible participants (high-risk stroke patients and their family members) were then drawn from 6 settings (1 gerontology department, 1 cardiovascular medicine department, 1 endocrinology department, and 3 local community groups) for item analysis.

The revised temporary scales were assessed in 616 eligible new participants (high-risk stroke patients and their family members) from 10 locations (2 gerontology departments, 2 cardiovascular medicine departments, 2 endocrinology departments, and 4 local community groups). The test-retest reliability was assessed by comparing the SPDBI scores of 86 additional participants (high-risk stroke patients and their family members) from 5 local community groups.

\section{Procedure}

A schematic representation of the study design is shown in Figure 1. The final SPDBI has two response formats depending on item phrasing. Both formats are based on a 5-point Likert scale of 1 (strongly serious or disagree) to 5 (nothing serious or highly agree). The overall SPDBI score is calculated by adding the item scores together. A higher score indicates a greater likelihood of pre-hospital delay.

\section{Stage 1: formulation of the draft SPDBI scale}

From a review of stroke scales, the Chinese guidelines for management and treatment of acute ischemic stroke (2010) [18], public education materials on strokes, and pre-hospital delay factors found in related studies, we formulated a prototype scale. We next verified the prototype by conducting semi-structured interviews in ten stroke patients. We asked them "what symptoms appeared when the stroke occurred, what did you think, and what did you do?" We recorded their answers and developed new items from them. Then, we hypothesized and developed a 63item, 6-factor (Factor 1: stroke warning signs, Factor 2: barriers to going to a doctor, Factor 3: symptom attributions, Factor 4: habitual response style, Factor 5: hospital chosen, Factor 6: vehicle use) draft SPDBI scale. All of the 


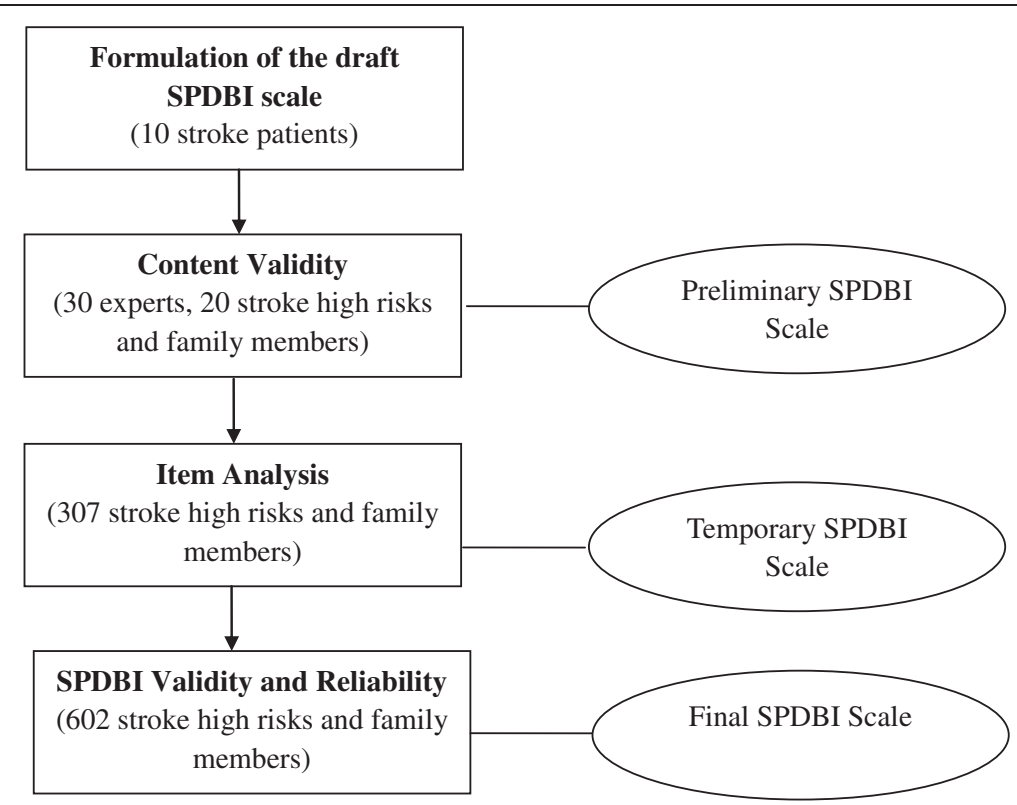

Figure 1 Schematic representation of the Stroke Pre-hospital Delay Behavior Intention (SPDBI) scale validation process.

items were presented in lay language so that the participants could clearly understand the meaning [13].

\section{Stage 2: content validity}

Two surveys were sent to experts. The first survey included an introductory letter, written consent forms, a questionnaire about the draft SPDBI scale, and the inquiry deadline. The authors visited the various clinical departments and personally disseminated the first survey questionnaire to the experts who met the recruitment requirements. A week later, the authors received the first survey results and analyzed them. Two weeks after dissemination the second survey began. This second survey included a summary of experts' advice from the first survey and the content validity value judgment-the correlation between item and the stroke pre-hospital delay behavior intention, from 4 (strongly related) to 1 (not related). The delivery method was the same as the first round. After the two surveys, 20 participants were asked to complete the preliminary scale to test the scale's comprehension and wording.

\section{Stage 3: item analysis}

Items in the preliminary scale that satisfied two or more of the following conditions were modified to formulate a temporary scale. (1) The variable's standard deviation was less than 0.75. (2) The degree of high-score and low-score group differentiation had no statistical significance at $p>0.05$. (3) Response analysis showed three or more ( $>2)$ ratings of the response rate that were less than $10 \%$. (4) Internal consistency analysis showed an item and total correlation coefficient score of less than 0.4. The remaining items were assessed by principal component factor analysis with varimax rotation to delete items that loaded $<0.4$ or cross-loaded (loaded on $\geq 2$ factors with values $\geq 0.4$ and with a difference of $<0.2$ between them) [19].

\section{Stage 4: SPDBI reliability and validity}

A reliability and validity study was then performed.

\section{Statistical analysis}

\section{Characteristics of the sample}

Descriptive statistics were used to analyze demographic information.

\section{Content validity}

Item content validity index in stage 2 is the number of expert choices of 3 and 4 divided by the total number of experts. Total content validity index (CVI) is the average of the item content validity index.

\section{Exploratory factor analysis (EFA)}

Principal Components Analysis (PCA) and Parallel analysis [20] were conducted. Parallel analysis is often recommended as the best method to assess the true number of factors [21].To assess the suitability of the factor solution, Bartlett's test of sphericity [22] should be significant, and the Kaiser-Meyer-Olkin (KMO) measure of sampling adequacy should be $>0.6$ [23]. The number of factors met the decision rules of (1) Kaiser's criterion (eigenvalues >1.0) [24] (2) inspection of the scree plot [25], and (3) Parallel analysis: Eigenvalues obtained from PCA with eigenvalues exceeding the values obtained from the 
corresponding random data set are retained [23],we conducted the parallel analysis online (http://ires.ku. edu/ smishra/parallelengine.htm).

\section{Confirmatory factory analysis (CFA)}

Confirmatory factor analysis (CFA) on the structural model was also performed in Stage 4; analysis of moment structures (Amos, 17.0) was used to test the model. The models' goodness of fit was evaluated using absolute and relative indices [26], including the root mean squared error of approximation (RMSEA; $<0.08$ acceptable), adjusted goodness of fit index (AGFI; >0.90 acceptable), Tucker-Lewis index (TLI), comparative fit index (CFI), and goodness of fit index (GFI) all >0.90 acceptable.

\section{Discriminate validity}

The assessment of discriminate validity was exploratory. The SPDBI scale total scores were compared among different groups. For the comparison between two groups, the $t$-test was used. One-way analysis of variance (ANOVA) for multiple comparisons was used where more than 2 groups were available and the data followed a normal distribution.

\section{Internal consistency reliability and test-retest reliability}

Nunnaly's criterion for satisfactory internal consistency reliability is a Cronbach's alpha of $\geq 0.7$ [27]. We also measured intraclass correlation coefficient (ICC) according to the following values: weak correlation $(\leq 0.4)$, moderate correlation (0.41-0.60), good correlation (0.61-0.80), and excellent correlation (0.81-1.00) [28].

\section{Results}

\section{Characteristics of the sample}

Experts' mean age was 39.5 years $( \pm 7.2$, range $=30-52)$. Over half had 10-20 years of experience (70\%) and the others had more than 20 years of experience (30\%). Approximately half had the professional title of nurse- or doctor-in-charge (43.3\%). Level of education was divided roughly equally between doctoral level (33.3\%), master's degrees $(36.7 \%)$, and bachelor's degrees (30\%). All were involved in work related to stroke pre-hospital delay: neurology (70\%), neurosurgery (20\%), and emergency departments (10\%), see Table 1 . Two experts were excluded in the second survey because of a business trip.

Five participants were excluded for various reasons in stage 3 , including participation refusal $(n=2)$, worsening patient condition $(\mathrm{n}=2)$, and incomplete information $(n=1)$, therefore, a total of 307 participants were included in item analysis. Similarly, fourteen participants were excluded in Stage 4 for different reasons, including lack of information $(n=4)$, refusal to participate $(n=3)$, worsening patient condition $(n=4)$, and other reasons $(n=3)$. The remaining 602 participants (Table 2) were
Table 1 Demographic characteristics of the first survey content validity sample

\begin{tabular}{lll}
\hline Characteristics & $\%$ & $\mathbf{n}$ \\
\hline Age, & 73.3 & 22 \\
$<45$ & 26.7 & 8 \\
$45-59$ & & \\
Years of experience & 70.0 & 21 \\
$10-20$ & 30.0 & 9 \\
$>20$ & & \\
Professional title & 3.3 & 1 \\
Senior nurse & 43.3 & 13 \\
Nurse-in-charge/doctor-in-charge & 23.3 & 7 \\
Co-chief nurse/associate doctor & 30.0 & 9 \\
Chief nurse/chief physician & & \\
Highest education completed & 30.0 & 9 \\
Bachelor & 36.7 & 11 \\
Master & 33.3 & 10 \\
Doctor & & 21 \\
Professional field & 70.0 & 6 \\
Neurology & 20.0 & 3 \\
Neurosurgery & 10.0 & \\
Emergency department & & \\
\hline
\end{tabular}

included in the final validity and reliability analysis (282 men and 320 women; 320 high-risk stroke patients and 282 family members; mean age 48.29 years $[ \pm 13.91]$ ).

\section{Content validity}

Data from the expert survey indicated that the content validity index (CVI) was 0.901. After the experts reached consensus, the preliminary SPDBI scale had 60 items and data showed that all 20 participants (10 high-risk stroke patients and 10 relatives) agreed that it was easy to understand with no difficulties reported.

\section{Item analysis}

After item analysis in stage 3, we kept 45 items. After the preliminary factor analysis on the test results, there were 7 items removed, formulating the 38-item temporary SPDBI scale.

\section{Exploratory factor analysis (EFA)}

The KMO test (0.897) showed adequate sampling adequacy, and Bartlett's test was significant ( $d f=703, p=0.000)$. Preliminary analyses produced a seven-factor model. A total of 11 items with a load $<0.4$ or with cross-load were deleted, PCA were finally revealed 5 factors, which was also supported by parallel analysis (Table 3). 27 items loaded substantially onto these 5 factors. The final produced factors (Table 4) included Factor 1, stroke warning 
Table 2 Demographic characteristics of Stage4 sample and comparison of Mean SPDBI Scores by 602 participants

\begin{tabular}{|c|c|c|c|c|}
\hline Characteristics & $\mathrm{N}(\%)$ & Mean(SD) & $P$ value & $95 \% \mathrm{Cl}$ \\
\hline \multicolumn{5}{|l|}{ Gender } \\
\hline Male & $282(46.8 \%)$ & 81.34(14.94) & 0.596 & $-3.15-1.73$ \\
\hline Female & $320(53.2 \%)$ & $82.05(15.48)$ & & \\
\hline \multicolumn{5}{|l|}{ Age } \\
\hline $18-85$ & $602(100 \%)$ & $81.72(15.22)$ & & \\
\hline$<40$ & $171(28.4 \%)$ & $80.99(14.16)$ & 0.242 & \\
\hline $40-60$ & $327(54.3 \%)$ & $82.64(15.08)$ & & \\
\hline$>60$ & 104(17.3\%) & $80.04(17.17)$ & & \\
\hline \multicolumn{5}{|l|}{ Habitual residence } \\
\hline City center & $445(73.9 \%)$ & $80.94(15.75)^{*}$ & $P<0.01$ & $1.62-8.50$ \\
\hline Suburb & $67(11.1 \%)$ & $81.16(13.75)$ & & \\
\hline Country & $90(15.0 \%)$ & $86.00(12.87)^{*}$ & & \\
\hline \multicolumn{5}{|l|}{ Classification } \\
\hline High-risk stroke patient & $320(53.26 \%)$ & $81.31(15.98)$ & 0.480 & $-3.32-1.56$ \\
\hline Family member & $282(46.8 \%)$ & $82.19(14.33)$ & & \\
\hline \multicolumn{5}{|c|}{ Family per capita monthly income (yuan) } \\
\hline$<1000$ & $67(11.1 \%)$ & $83.22(13.92)$ & $P<0.05$ & $0.57-5.84$ \\
\hline $1000-2000$ & $212(35.2 \%)$ & $83.47(15.35)^{*}$ & & \\
\hline$>2000$ & $323(53.7 \%)$ & $80.26(15.29)^{*}$ & & \\
\hline \multicolumn{5}{|l|}{ Physical examination } \\
\hline More than once per year & 195(32.4\%) & $80.11(17.25)^{*}$ & $P<0.05$ & $0.27-6.32$ \\
\hline A few years at a time & $206(34.2 \%)$ & $83.36(14.53)^{*}$ & & \\
\hline Never & $201(33.4 \%)$ & $81.61(13.64)$ & & \\
\hline \multicolumn{5}{|l|}{ Self-report stroke knowledge } \\
\hline Received stroke knowledge education & $76(12.6 \%)$ & $78.92(18.96)^{*}$ & $P<0.05$ & $0.28-8.41$ \\
\hline Saw someone suffering from stroke & $289(48.0 \%)$ & $81.30(15.27)$ & & \\
\hline Know nothing about stroke & $237(39.4 \%)$ & $83.13(13.66)^{*}$ & & \\
\hline
\end{tabular}

Notes: $\mathrm{N}=602$. There are statistically significant differences between the groups with "**.

signs ( 9 items) by which participants judge the severity of the stroke symptoms listed; Factor 2, non-treatment justification (8 items )describing alternative explanations and not receiving treatment; Factor 3, symptom attributions (4 items) or participants' analyses of the stroke symptom causes; Factor 4, habitual response style (3 items) to determine whether the participants usually responded to medical symptoms immediately; and Factor 5, emergency system use (3 items) regarding the likelihood of the

Table 3 The results of parallel analysis

\begin{tabular}{lll}
\hline Eigen values & Means & Percentile \\
\hline 7.479 & 1.511189 & 1.569466 \\
4.484 & 1.445252 & 1.486443 \\
2.169 & 1.405245 & 1.437552 \\
1.745 & 1.366892 & 1.395265 \\
1.343 & 1.332469 & 1.357914 \\
\hline
\end{tabular}

participant's choice of hospitalization and transport. The factor scree plot also showed a suitable 5-factor solution; these factors contributed to $53.447 \%$ of the variance.

\section{Confirmatory factory analysis (CFA)}

Table 5 shows that the degree of fit and stability of the structural model are good to excellent.

\section{Discriminate validity}

The SPDBI's total score had statistically significant differences in the following aspects: habitual residence, family per capita monthly income, physical examination, and self-report stroke knowledge $(p<0.05)$ (Table 2). However, age, classification, and gender (Table 2) were not associated with the total score. 
Table 4 Factor structure of the final version of SPDBI

\section{Sub-domain 1: stroke warning signs}

V1.Inconsistent in thinking and language; answers to the problems such as time and

V3.Can be awakened and was able to answer simple questions, but slowly, then continued

to sleep when stimulation stopped

V4.Weakness, heaviness, or numbness on one side of the limb

V5.Vertigo (see rotation), blacked out

V7.Double vision on one side of the eyes

V8.Clear pronunciation, but of incorrect and ambiguous words

\section{Sub-domain 2: non-treatment justification}

V10.Don't go to the hospital because the results are the same whether or not you go 0.793

V11.Don't go to the hospital because it is too much trouble 0.783

V12.Don't go to the hospital because worried about added burden to family. 0.780

V13.Don't go to the hospital because symptoms are from being old and weak 0.762

V14.Don't go to the hospital because body is usually ok and symptoms are no big deal. 0.754

V15.Patient will soon recover and symptoms are nothing important 0.720

V16.Patient will first rest and see how they feel since the weather is bad 0.641

V17.I will wait since there is no one around to help me 0.613

\section{Sub-domain 3: symptom attributions}

V18.Sudden weakness, heaviness, or numbness on one side of the limb is just recent tiredness 0.671

V19.Sudden blurred vision in one or both eyes is from excessive eye use 0.661

V20.Weakness, clumsiness on one side of the limb in the morning, because pressure to stay in bed 0.644

V21.Sudden headache and dizziness are caused by a cold 0.530

Sub-domain 4: habitual response style

V22.My first thought is to have a rest at onset of symptoms 0.692

V23.My first thought is to take some medicine at onset of symptoms 0.768

V24.If my symptoms don't improve(or worsen),then I will go to the hospital 0.613

\section{Sub-domain 5: emergency system use}

V25.Don't call an ambulance because of the high cost 0.721

V26.I can't think to call an ambulance at first 0.683

V27.I chose a Chinese medicine hospital suggested by an acquaintance 0.606

Table 5 Fit indices for the model

\begin{tabular}{lllllll}
\hline & $\boldsymbol{x}^{2} / \mathbf{d f}$ & GFI & AGFI & RMSEA & CFI & TLI \\
\hline Model & 2.286 & 0.917 & 0.900 & 0.046 & 0.924 & 0.915 \\
Fit criteria & $<5$ & $>0.9$ & $>0.8$ & $<0.08$ & $>0.9$ & $>0.9$
\end{tabular}

GFI, goodness-of-fit index; AGFI, adjusted goodness-of-fit index; RMSEA, root mean square error of approximation; $\mathrm{CFI}$, comparative fit index; $\mathrm{TLI}$ Tucker-Lewis index.
Internal consistency reliability and test-retest reliability The Cronbach's alpha is 0.808 . Test-retest reliability for total scale was good with ICC $=0.797$, and for sub-domains ICC $=0.676-0.819$ (Table 6).

\section{Discussion}

Behavioral intention refers to the subjective probability of an individual engaging in a particular behavior and reflects the will an individual adopts for a particular behavior [29]. Our research defines pre-hospital delay in behavioral 
Table 6 The SPDBI ICCs across the two weeks of the test-retest interval

\begin{tabular}{llll}
\hline Sub-domains & Number of items & ICC & $\mathbf{9 5 \% ~ C l}$ \\
\hline Sub-domain 1 & 9 & 0.777 & $0.658-0.855$ \\
Sub-domain 2 & 8 & 0.819 & $0.722-0.882$ \\
Sub-domain 3 & 4 & 0.742 & $0.605-0.832$ \\
Sub-domain 4 & 3 & 0.714 & $0.562-0.814$ \\
Sub-domain 5 & 3 & 0.676 & $0.503-0.789$ \\
Total & 27 & 0.797 & $0.688-0.867$ \\
\hline
\end{tabular}

intention as delayed treatment from symptom appearance to reaching a hospital with appropriate interventions. We developed a scale that includes not only the severity of stroke warning signs but also specific items on how the participants would think about or react to stroke signs. The SPDBI may indeed be particularly suitable for use in behavior intention programs, which recognize the potential impact of participants' cognition and behavior on stroke pre-hospital delay. First, we can determine which predictors of the scale are more likely to lead to participants' pre-hospital delay. Second, we could use this tool to select those at high risk of stroke and their family members who scored higher, and provide them with systematic health education intervention. The more targeted such health education is, the more likely participants are to cooperate and save social resources at the same time. Third, by applying this scale before and after health education, we can compare the scores before and after the intervention with the pre-hospital delay time length or rate of thrombolysis, using tracing methods to verify the exactness of the scale prediction.

The SPDBI scale has good reliability and validity. We involved experts in neurology, neurosurgery, and emergency departments to test the content validity. All of these experts are in close contact with stroke patients, and communicate with patients in the process of admits, treatment, or care, so they have significant understanding of the degree of pre-hospital delay and factors influencing patients. In addition, the experts were distributed among different units and did not know the names of other experts and their departments, so we avoided communication between experts. More importantly, we also selected 20 high-risk participants and their close family members to test the wording and understanding. Comprehensively, the content validity of this research is good and, after further item revision, potentially improvable. Both exploratory and confirmatory factor analyses are appropriately used when a hypothesized measurement model is evaluated [30]. The sample size should be at least 10-15 individuals per variable for an exploratory factor analysis [30]. Our sample size was large enough for the analyses. EFA shows 5 conceptually clear and psychometrically robust sub-domains and CFA suggests that the fit of the model's structure and stability is good. Our result for Cronbach's alpha is high. The stability of the total scale is good (ICC $>0.7$ ).

The discriminate validity evidence is that the scale can distinguish between groups of differences, the evidences are as follows:

First, the result showed that the group with poorer self-report stroke knowledge before testing had a higher SPDBI score than the group with better knowledge. As the SPDBI scale score predicted, we found similar results to other studies, i.e., knowing someone who had suffered a stroke was not associated with shorter pre-hospital delay, [31] and stroke knowledge received from other information sources was associated with shorter pre-hospital delay $[32,33]$.

Second, the SPDBI scale could identify different total scores between groups with different family per capita monthly income and groups with different habitual residence. The groups with lower family per capita monthly income scored higher. This may be because although total public expenditure on health insurance in China has been rising steadily since 2006, the level of satisfaction with the health care system has remained low. There are several out-of-pocket medical costs [34], meaning that lower income people may be more likely to delay hospitalization. Besides, the groups with habitual residence in country scored higher, this may be because of greater distances from the hospital for treatment; ambulance arrival times remain varied across China [35] and EMS systems are absent in most Chinese areas [36].

Third, the participants who have fewer physical examinations will potentially delay hospitalization. One reason could be that people understand their health condition through physical examinations, which could help them to take timely and preventive actions. The other reason might be that doctors and nurses were regarded as the best source of stroke information [37], and people were encouraged to take a positive attitude when facing health problems in the process of physical examinations. Furthermore, physical examinations promoted access to health knowledge for people who prefer not to be subjected to medical checkups; this is especially true of the free physical examination by some work units in China.

Fourth, there were no significant difference in SPDBI subscale and total scores by gender, our analysis predicted gender was not a significant factor associated with prehospital delays in the presentation of acute stroke in urban China (Table 2). Jin et al. [5] found a similar result. However, data regarding gender differences in other countries for knowledge about stroke symptoms and correct behavior are conflicting. Women had better knowledge of stroke symptoms and faster arrival to the hospital in some studies $[37,38]$, but poorer knowledge of stroke symptoms and later arrival to the hospital among older stroke patients 
[16], and were not associated with stroke presentation or management in another study [39].One explanation for our result might be the Chinese traditional culture of promoting family harmony. Most of our participants (93.2\%), even the aged, live with their family members, thus reducing the possibility of older persons (especially older women) living alone, and are more likely to fall requiring help quickly [16]. Even so, we need to note that women have a greater risk of dying from stroke owing to their longer life expectancy [39].

We evaluated the instrument not only for use with groups of less healthy individuals (those with a high risk of stroke) but also their close family members. Important findings from other studies show that more than 80\% [40] of patients had their strokes at home and these patients experienced longer delays. However, the person seeking medical help was rarely the patient himself. Patients only called an ambulance for themselves $3 \%$ of the time, frequently relying on family members instead. Indeed, in $37-68 \%$ of the cases, the decision (use of EMS) is made by a family member $[5,40]$. Any program aimed at increasing stroke awareness needs to target a broad community audience. Our study therefore highlights the urgent need to evaluate the high-risk stroke patients' family members. The authors have compared the scores of high-risk patients and their family members, and an independent $t$-test found that their scores had no significant differences $(p>0.05)$. Family members should also know the seriousness of stroke symptoms and the benefits of immediately going to a hospital for treatment if a stroke occurs.

The mean of the total scale score was $81.72 \pm 15.22$. If we consider this mean score as a cut-off point, then scores that are higher than this point predict a higher possibility of pre-hospital delay, and special attention would need to be paid to those in this group. In our results, 327 of 602 (54.3\%) participants' total scores surpassed the cut-off. This point is slightly lower than the $63 \%$ of stroke patients with pre-hospital delay reported by Jin et al. [5]. This may be because more than $80 \%$ of our participants are from cities where medical treatment is more convenient and the higher incidence of stroke in northeastern China may increase opportunities for health education.

\section{Limitations/future direction}

There were some limitations in our study. First, we could not evaluate the psychometric properties of convergent validity, because we have not found other instruments that predict stroke pre-hospital delay. Furthermore, the scale is a Chinese version (Additional file 1). In China, people may face problems such as "high cost for an ambulance" or "prefer a Chinese medicine hospital," which may not generalize to other countries. Further cross-cultural revisions and validation are needed in the international application of the scale in the future. Third, we need to continue working on ways to portray "warning sign" symptoms in the sub-domain more realistically, perhaps through the use of pictures or multimedia technology, as this would increase the predictive value of the scale. Finally, more than $80 \%(512 / 602)$ of our participants are citizens of Harbin and responses to acute strokes could not, therefore, be generalized to rural areas or remote regions of China. It would also be important to evaluate the instrument for use with individuals from those areas in a future study.

\section{Conclusion}

In summary, the present study has rigorously developed and validated the SPDBI scale, providing scores with good reliability and validity. This scale assesses high-risk stroke patients and their family members' possibility of prehospital delay if stroke happens, and might help to decrease stroke pre-hospital delay. Thus, it would greatly facilitate more targeted public education efforts in China.

\section{Additional file}

Additional file 1: The English and Chinese version of SPDBI scale, and the electronic ethic prove.

\section{Competing interests}

The authors declare that they have no competing interests.

\section{Authors' contributions}

QZ, LY, and QZ contributed equally to this work. All authors read and approved the final manuscript.

\section{Acknowledgements}

We sincerely thank the 30 experts for their cooperation in participating in the study (Yulan Zhu, Chunjiang Yu, Xiangnan Huang, Benping Zhang, Yonghua Jin, Yanmei Zhu, Qingcheng Liang, Lihua Wang, Chunxiao Yang, Xiaobo Yan, Yun Wu ,Jing Man,Yajing Li, Xin Liu, Zhaojun Liu, Meichun Cheng, Hongmei Zhu, Shasha Du, Shujie Shi, Dong Pang, Lina Du from 6 Neurology department; Jianwen Wang, Haicheng Yang, Shaoshan Hu, Shihong Zhao, Qiuli Bao, Minghuan Zhang from 3 Neurosurgery department; Dan Cui, Dongmei Li, Jie Zhan from Emergency department) associated with the content validity. The authors also wish to thank the participants for their involvement in this study.

\section{Funding}

This project was funded by the National Natural Science Foundation of China (no. 81273194).

\section{Author details}

${ }^{1}$ School of Nursing, The 2nd Affiliated Hospital of Harbin Medical University, Harbin Medical University, 246 Xuefu Road, Harbin, HeiLongJiang Province 150086, China. ${ }^{2}$ Department of Nephrology, The 2nd Affiliated Hospital of Harbin Medical University, Harbin Medical University, Harbin, HeiLong Jiang Province 150086, China.

Received: 26 March 2014 Accepted: 12 November 2014

Published online: 29 November 2014 


\section{References}

1. Meyers PM, Schumacher HC, Connolly ES Jr, Heyer EJ, Gray WA, Higashida RT: Current status of endovascular stroke treatment. Circulation 2011, 123(22):2591-2601.

2. Hacke W, Kaste M, Bluhmki E, Brozman M, Dávalos A, Guidetti D, Larrue V, Lees KR, Medeghri Z, Machnig T, Schneider D, Kummer R, Wahlgren N, Toni D: Thrombolysis with alteplase 3 to 4.5 hours after acute ischemic stroke. N Engl J Med 2008, 359(13):1317-1329.

3. Wahlgren N, Ahmed N, Davalos A, Hacke W, Millan M, Muir K, Roine RO, Toni D, Lees KR: Thrombolysis with alteplase 3-4.5 $\mathrm{h}$ after acute ischaemic stroke (SITS-ISTR): an observational study. Lancet 2008, 372(9646):1303-1309.

4. Anderson CS, Huang Y, Wang JG, Arima H, Neal B, Peng B, Heeley E, Skulina C, Parsons MW, Kim JS, Tao QL, Li YC, Jiang JD, Tai LW, Zhang JL, Xu E, Cheng Y, Heritier S, Morgenstern LB, Chalmers J: Intensive blood pressure reduction in acute cerebral haemorrhage trial (INTERACT): a randomised pilot trial. Lancet Neurol 2008, 7(5):391-399.

5. Jin H, Zhu S, Wei JW, Wang J, Liu M, Wu Y, Wong LK, Cheng Y, Xu E, Yang Q, Anderson CS, Huang YN: Factors associated with prehospital delays in the presentation of acute stroke in urban China. Stroke 2012, 43(2):362-370.

6. Evenson KR, Foraker RE, Morris DL, Rosamond WD: A comprehensive review of prehospital and in-hospital delay times in acute stroke care. Int I Stroke 2009, 4(3):187-199.

7. Shah M, Makinde KA, Thomas P: Cognitive and behavioral aspects affecting early referral of acute stroke patients to hospital. I Stroke Cerebrovasc Dis 2007, 16(2):71-76.

8. Kidwell CS, Starkman S, Eckstein M, Weems K, Saver JL: Identifying stroke in the field: prospective validation of the Los Angeles Prehospital Stroke Screen (LAPSS). Stroke 2000, 31(1):71-76.

9. Kothari RU, Pancioli A, Liu T, Brott T, Broderick J: Cincinnati prehospital stroke scale: reproducibility and validity. Ann Emerg Med 1999, 33(4):373-378

10. Harbison J, Hossain O, Jenkinson D, Davis J, Louw SJ, Ford GA: Diagnostic accuracy of stroke referrals from primary care, emergency room physicians, and ambulance staff using the face arm speech test. Stroke 2002, 34(1):71-76.

11. Mikulik R, Bunt L, Hrdlicka D, Dusek L, Vaclavik D, Kryza J: Calling 911 in response to stroke: a nationwide study assessing definitive individual behavior. Stroke 2008, 39(6):1844-1849.

12. Lindley Rl: Improving onset to needle time: knowledge is not enough. Stroke 2008, 39(6):1667.

13. Billings-Gagliardi S, Mazor KM: Development and validation of the stroke action test. Stroke 2005, 36(5):1035-1039.

14. Kleindorfer DO, Lindsell CJ, Broderick JP, Flaherty ML, Woo D, Ewing I, Schmit P, Moomaw C, Alwell K, Pancioli A, Jauch E, Khoury J, Miller R, Schneider A, Kisselaet BM: Community socioeconomic status and prehospital times in acute stroke and transient ischemic attack: do poorer patients have longer delays from 911 call to the emergency department? Stroke 2006, 37(6):1508-1513.

15. Chen C-H, Huang P, Yang Y-H, Liu C-K, Lin T-J, Lin R-T: Pre-hospital and in-hospital delays after onset of acute ischemic stroke-a hospital-based study in southern Taiwan. Kaohsiung J Med Sci 2007, 23(11):552-559.

16. Foerch C, Misselwitz B, Humpich M, Steinmetz H, Neumann-Haefelin T, Sitzer M: Sex disparity in the access of elderly patients to acute stroke care. Stroke 2007, 38(7):2123-2126.

17. Kozera G, Chwojnicki K, Gojska-Grymajlo A, Gasecki D, Schminke U, Nyka WM: Pre-hospital delays and intravenous thrombolysis in urban and rural areas. Acta Neurol Scand 2012, 126(3):171-177.

18. Wang YJ, Zhang SM, Zhang L, Wang CX, Dong Q, Gao S, Huang RX, Huang YN, LV CZ, Liu M: Chinese guidelines for the secondary prevention of ischemic stroke and transient ischemic attack 2010. CNS Neurosci Ther 2012, 18(2):93-101.

19. Hilari K, Byng S, Lamping DL, Smith SC: Stroke and Aphasia Quality of Life Scale-39 (SAQOL-39): evaluation of acceptability, reliability, and validity. Stroke 2003, 34(8):1944-1950.

20. Basto M, Pereira JM: An SPSS R-menu for ordinal factor analysis. J Stat Softw 2012, 46(4):1-29. http://www.jstatsoft.org/v46/i04/paper.

21. Lance $C E$, Butts $M M$, Michels $L C$ : The sources of four commonly reported cutoff criteria: what Did they really Say?". Organ Res Methods 2006, 9(2):202-220.
22. Ba MS: A note on multiplying factors for various chi square approximations. J Royal Stat Soc 1954, 16(Series B):296-298.

23. Mills RJ, Young CA, Pallant JF, Tennant A: Development of a patient reported outcome scale for fatigue in multiple sclerosis: the Neurological Fatigue Index (NFI-MS). Health Qual Life Outcomes 2010, 8:22

24. Ka HF: The application of electronic computers to factor analysis. Educ Psychol Meas 1960, 20:141

25. Ca RB: The scree test for the number of factors. Multi Behav Res 1966, 1:245-276.

26. Sun T, Zhao XW, Yang LB, Fan LH: The impact of psychological capital on job embeddedness and job performance among nurses: a structural equation approach. J Adv Nurs 2012, 68(1):69-79.

27. Anderson C, Laubscher S, Burns R: Validation of the Short Form 36 (SF-36) health survey questionnaire among stroke patients. Stroke 1996 27(10):1812-1816.

28. Landis JKG: The measurement of observer agreement for catgegorical data. Biometrics 1977, 33:159-174.

29. Ajzen I: The theory of planned behaviour: reactions and reflections. Psychol Health 2011, 26(9):1113-1127.

30. Ekback M, Benzein E, Lindberg M, Arestedt $K$ : The Swedish version of the multidimensional scale of perceived social support (MSPSS)-a psychometric evaluation study in women with hirsutism and nursing students. Health Qual Life Outcomes 2013, 11(168):1477-7525.

31. Rosamond WD, Gorton RA, Hinn AR, Hohenhaus SM, Morris DL: Rapid response to stroke symptoms: the Delay in Accessing Stroke Healthcare (DASH) study. Acad Emerg Med 1998, 5(1):45-51.

32. Moser DK, McKinley S, Dracup K, Chung ML: Gender differences in reasons patients delay in seeking treatment for acute myocardial infarction symptoms. Patient Educ Couns 2005, 56(1):45-54

33. Moser DK, Kimble LP, Alberts MJ, Alonzo A, Croft JB, Dracup K, Evenson KR, Go AS, Hand MM, Kothari RU, Mensah GA, Morris DL, Pancioli AM, Riegel B, Zerwic JJ: Reducing delay in seeking treatment by patients with acute coronary syndrome and stroke: a scientific statement from the American Heart Association Council on cardiovascular nursing and stroke council. Circulation 2006, 114(2):168-182.

34. Cao Q, Shi L, Wang H, Dong K: Report from China: health insurance in China-evolution, current status, and challenges. Int J Health Serv 2012, 42(2):177-195.

35. Hung KK, Cheung CS, Rainer TH, Graham CA: EMS systems in China Resuscitation 2009, 80(7):732-735.

36. Kamran S, Bener AB, Deleu D, Khoja W, Jumma M, Al Shubali A, Inshashi J, Sharouqi I, Al Khabouri J: The level of awareness of stroke risk factors and symptoms in the gulf cooperation council countries: gulf cooperation council stroke awareness study. Neuroepidemiology 2007, 29(3-4):235-242.

37. Stroebele N, Muller-Riemenschneider F, Nolte CH, Muller-Nordhorn J, Bockelbrink A, Willich SN: Knowledge of risk factors, and warning signs of stroke: a systematic review from a gender perspective. Int I Stroke 2011, 6(1):60-66

38. Nolte CH, Rossnagel $\mathrm{K}$, Jungehuelsing GJ, Muller-Nordhorn J, Roll S, Reich A, Willich SN, Villringer A: Gender differences in knowledge of stroke in patients with atrial fibrillation. Prev Med 2005, 41(1):226-231.

39. Kapral MK, Fang J, Hill MD, Silver F, Richards J, Jaigobin C, Cheung AM: Sex differences in stroke care and outcomes: results from the registry of the Canadian stroke network. Stroke 2005, 36(4):809-814.

40. Teuschl Y, Brainin M, Stroke education: Discrepancies among factors influencing prehospital delay and stroke knowledge. Int J Stroke 2010, 5(3):187-208.

doi:10.1186/s12955-014-0170-8

Cite this article as: Zhao et al: Instrument development and validation of the stroke pre-hospital delay behavior intention scale in a Chinese urban population. Health and Quality of Life Outcomes 2014 12:170. 